\title{
Vorinostat-induced autophagy switches from a death-promoting to a cytoprotective signal to drive acquired resistance
}

\author{
D Dupéré-Richer ${ }^{1}$, M Kinal ${ }^{1}$, V Ménasché ${ }^{1}$, TH Nielsen ${ }^{1}$, S del Rincon ${ }^{1}$, F Pettersson ${ }^{1}$ and WH Miller $\mathrm{Jr}^{\star, 1}$
}

Histone deacetylase inhibitors (HDACi) have shown promising activity against hematological malignancies in clinical trials and have led to the approval of vorinostat for the treatment of cutaneous T-cell lymphoma. However, de novo or acquired resistance to HDACi therapy is inevitable, and their molecular mechanisms are still unclear. To gain insight into HDACi resistance, we developed vorinostat-resistant clones from the hematological cell lines U937 and SUDHL6. Although cross-resistant to some but not all $\mathrm{HDACi}$, the resistant cell lines exhibit dramatically increased sensitivity toward chloroquine, an inhibitor of autophagy. Consistent with this, resistant cells growing in vorinostat show increased autophagy. Inhibition of autophagy in vorinostatresistant U937 cells by knockdown of Beclin-1 or Lamp-2 (lysosome-associated membrane protein 2) restores sensitivity to vorinostat. Interestingly, autophagy is also activated in parental U937 cells by de novo treatment with vorinostat. However, in contrast to the resistant cells, inhibition of autophagy decreases sensitivity to vorinostat. These results indicate that autophagy can switch from a proapoptotic signal to a prosurvival function driving acquired resistance. Moreover, inducers of autophagy (such as mammalian target of rapamycin inhibitors) synergize with vorinostat to induce cell death in parental cells, whereas the resistant cells remain insensitive. These data highlight the complexity of the design of combination strategies using modulators of autophagy and $\mathrm{HDACi}$ for the treatment of hematological malignancies.

Cell Death and Disease (2013) 4, e486; doi:10.1038/cddis.2012.210; published online 7 February 2013

Subject Category: Cancer

Histone deacetylase inhibitors (HDACi) induce apoptosis, differentiation and growth arrest of cancer cells, whereas normal cells are relatively insensitive. HDACi now encompass different structural classes, including hydroxamate, fatty acids and benzamides. The hydroxamates vorinostat (suberoylanilide hydroxamic acid, Zolinza) and romidepsin were approved for the treatment of cutaneous T-cell lymphoma. ${ }^{1}$

HDACs catalyze the removal of acetyl groups from both histone and non-histone proteins. The antitumor action of HDACi was initially correlated with the reactivation of tumor suppressor genes potentially silenced in tumor cells through epigenetic mechanisms. ${ }^{2}$ However, Choudhary et al. ${ }^{3}$ identified 1750 proteins acetylated by vorinostat that regulate various pathways. Indeed, suggested mechanisms of action for their antineoplastic activity include: induction of DNA damage, ${ }^{4}$ ROS production, ${ }^{5}$ modulation of the cell cycle, ${ }^{6}$ modification of the turnover of oncogenes, ${ }^{7}$ among others. Therefore, the mechanism by which HDACi induce apoptosis of cancer cells is still far from defined.

Clinical studies with HDACi have shown interesting results in hematological malignancies. Seventeen percent of participants in a phase 1 study investigating vorinostat in patients with refractory MDS or leukemia showed clinical improvement. ${ }^{8}$ A phase 2 study of vorinostat in non-Hodgkin's lym- phoma reported complete responses in 4 out of 17 patients. ${ }^{9}$ However, as with many cancer therapies, de novo resistance is common and acquired resistance inevitably follows sensitivity. An understanding of the molecular mechanisms underlying resistance to HDACi may help identify predictive biomarkers for response to HDACi therapy. Proposed mechanisms of resistance to HDACi include increased antioxidant capacity of the cell, ${ }^{8,10,11}$ alteration of the drug target, ${ }^{12,13}$ deregulation of proapoptotic and prosurvival gene expres$\operatorname{sion}^{14,15}$ and induction or suppression of autophagy. ${ }^{16}$

Autophagy is a tightly regulated process involved in homeostasis, which helps maintain a balance between the synthesis, degradation and subsequent recycling of proteins. The role of autophagy in anticancer therapy is still under debate. ${ }^{17}$ Although some studies suggest that autophagy may function as a stress response helping to promote cell survival, others show that increased autophagy leads to apoptosis. ${ }^{18}$

To gain insight into acquired HDACi resistance in hematological malignancies, we developed vorinostat-resistant clones from the monocytic-like histiocytic lymphoma cell line U937 and the diffuse large B-cell lymphoma (DLBCL) SUDHL6. Interestingly, we found that the resistant cells exhibit increased sensitivity toward chloroquine (CQ), an inhibitor of autophagy. We therefore investigated the role of

\footnotetext{
${ }^{1}$ Lady Davis Institute for Medical Research, Segal Cancer Center, Jewish General Hospital, McGill University, Montreal, Quebec, Canada

${ }^{*}$ Corresponding author: Dr WH Miller Jr, Lady Davis Institute for Medical Research, Segal Cancer Centre, Jewish General Hospital, McGill University, 3755 Côte Ste-Catherine Road, Montreal, Quebec H3T 1E2, Canada. Tel: + 5143408222 ext. 4365; Fax: + 514340 7574; E-mail: wmiller@Idi.jgh.mcgill.ca Presented in abstract form at the American Association for Cancer Research Annual Meeting in Chicago, IL, April 3, 2012.

Keywords: histone deacetylase inhibitor; autophagy; lymphoma; resistance

Abbreviations: HDACi, histone deacetylase inhibitors; $\mathrm{CQ}$, chloroquine; PI, propidium iodide; TEM, transmission electron microscopy; Lamp-2, lysosome-associated membrane protein 2; LC3, microtubule-associated protein light-chain 3; mTOR, mammalian target of rapamycin

Received 12.07.12; revised 18.11.12; accepted 29.11.12; Edited by G Ciliberto
} 
autophagy in resistant cells and in parental cells after shortterm exposure to vorinostat. We show that activation of autophagy promotes apoptosis in vorinostat-treated U937 parental cells, while even greater activation of autophagy in vorinostat-resistant clones is necessary to protect the cells from apoptosis and maintain the resistant phenotype.

\section{Results}

To derive a vorinostat-resistant cell line from the U937 cell line, we first developed a polyclonal population capable of growing in $2 \mu \mathrm{M}$ vorinostat, using a dose escalation protocol. Then, monoclonal populations of resistant cells were isolated by limiting dilution in the presence of $2 \mu \mathrm{M}$ vorinostat. We thus obtained multiple resistant cell populations, and we focused our investigations on one representative clonal population, U937-B8. The U937-B8 subline is continuously maintained in $2 \mu \mathrm{M}$ vorinostat to preserve the resistant phenotype, where it proliferates without evidence of apoptosis. In parental U937 cells, exposure to $1.5 \mu \mathrm{M}$ vorinostat leads to approximately $80 \%$ cell death within $48 \mathrm{~h}$, as assessed by DNA fragmentation using propidium iodide (PI) staining (Figure 1a). U937-B8 cells exhibit a slower growth rate compared with U937 cells, as represented by a higher doubling time (Figure 1b). After removal of vorinostat from the culture media for 1 week, the doubling time of U937-B8 cells decreases (Figure 1b), but cells maintain resistance to vorinostat-induced growth inhibition (Figure 1c), apoptosis (Figure 1a) and caspase $3 / 7$ activation (Figure 1d). However, U937-B8 cells grown without vorinostat for more than 3 weeks reacquire partial sensitivity towards vorinostat (Figure 1a), and their doubling time becomes equivalent to U937 cells (Figure 1b). Similar results were obtained from another vorinostat-resistant monoclonal cell line (U937-E5) (Supplementary Figures S1A and B).

We then looked at the toxicity of different HDACi in U937-B8 cells 1 week after removal of vorinostat from the culture media, by measuring cell death by PI staining. U937-B8 cells show cross-resistance to the proapoptotic effects of the hydroxamic acid pan-HDACi LBH589 and the HDAC6specific inhibitor tubastatin (Figure 1e), associated with the loss of caspase 3/7 activation (data not shown). Conversely, the class I-specific HDACi MGCD0103 induces cell death to the same extent in U937 and U937-B8 (Figure 1e). Similar results were obtained in the resistant subclone U937-E5 (Supplementary Figure $\mathrm{S1C}$ ). These results suggest that MGCD0103 toxicity in these cells may be mediated by a different mechanism than LBH589, tubastatin and vorinostat. Deregulation of the drug target is a common mechanism of resistance to targeted therapy. We therefore assessed the protein level of the main HDAC isoforms targeted by the HDACi tested, using western blotting, in parental and resistant cells, but did not detect any significant differences (Figure 1f).

Vorinostat has been proposed to induce apoptosis in the G0-G1 phase of the cell cycle by inducing the cyclindependent kinase inhibitor p21 in U937 cells. ${ }^{6}$ We observed an arrest in the G1 phase of the cell cycle, when U937 cells were exposed to $1.5 \mu \mathrm{M}$ vorinostat (Figure $1 \mathrm{~g}$ ). However, as we have shown previously, ${ }^{19}$ exposure to higher concentration of vorinostat, that is, $2 \mu \mathrm{M}$, leads to a block in the G2-M phase before apoptosis is detected (Figure 1g).
U937-B8 cells that have been cultured in the absence of drug for 1 week and subsequently exposed to 1.5 or $2 \mu \mathrm{M}$ vorinostat arrest in the G1 phase, but do not undergo apoptosis (Figure 1a, c, d and g).

To begin to define pathways involved in resistance to vorinostat, we evaluated the lethal dose $(L D)_{50}$ of various drugs with different mechanisms of action in U937-B8 versus their U937 parental counterpart (Table 1). LD 50 was calculated by measuring apoptosis using PI staining after $48 \mathrm{~h}$ exposure to drug. Although the growth rate of U937-B8 cells is slower than U937 cells (Figure 1b), the cells have an equivalent $L D_{50}$ for the microtubule-stabilizing agent taxol. U937-B8 cells were slightly more resistant to the DNA-damaging agent cisplatin and doxorubicin, and to the inducer of reactive oxygen species arsenic trioxide. In contrast, U937-B8 cells have a substantially lower LD $_{50}$ for chloroquine (CQ), an inhibitor of autophagy. The sensitivity to $\mathrm{CQ}$ decreases progressively with time after the removal of vorinostat from the culture media. We therefore hypothesized that autophagy is induced by the presence of vorinostat and that it might act as a prosurvival pathway to escape the cytotoxic effects of vorinostat. Indeed, we observed that $\mathrm{CQ}$ has a strong toxic effect in U937-B8 cells grown in vorinostat, as shown by increased levels of cell death and caspase 3/7 activation. This effect decreases 1 week after vorinostat has been removed from the culture media (Figure $2 a$ and b).

We subsequently tested whether $C Q$ also enhances cell death in the parental U937 cells exposed to vorinostat. Surprisingly, cotreatment with the same concentrations of $\mathrm{CQ}$ has a protective effect on vorinostat-induced cell death and caspase 3/7 activation in U937 cells (Figure 2c-d). Cell cycle analyses are shown in Supplementary Figure S2A and B. These results suggest that autophagy acts as a mediator of cell death during vorinostat initial exposure, but mediates cell survival following chronic exposure.

We next tested whether autophagy is increased in U937-B8 cells and if vorinostat induces autophagy in parental U937 cells. CQ accumulates selectively inside lysosomes, raising intralysosomal $\mathrm{pH}$, which blocks degradation of the lysosomal content. ${ }^{20}$ Because autophagosomes, and their content, are degraded by lysosomes, exposure to $\mathrm{CQ}$ induces an accumulation of intact autophagosomes. Using transmission electron microscopy (TEM), we observed dramatic subcellular morphological differences between U937-B8 cells and their parental counterparts. U937-B8 cells show a marked increase in large, clear autophagosomes following $18 \mathrm{~h}$ exposure to $25 \mu \mathrm{M} \mathrm{CQ}$, whereas smaller and fewer autophagosomes are observed in U937 cells exposed to the same conditions (Figure 3a), indicating increased basal autophagy in U937-B8 cells. In U937 cells exposed to vorinostat, cotreatment with $\mathrm{CQ}$ induces accumulation of small electron-dense autophagosomes, unlike those seen in U937-B8 cells (Figure 3a, bottom). Thus, although acute vorinostat exposure induces autophagy in U937 cells, the morphology of the autophagosomes suggests a higher level of basal autophagy induced by chronic exposure to vorinostat in the U937-B8-resistant cells.

To confirm these results, we assessed the levels of key proteins involved in the autophagic process in parental and resistant cell lines. Autophagy is routinely assessed by observing the marker LC3 (microtubule-associated 


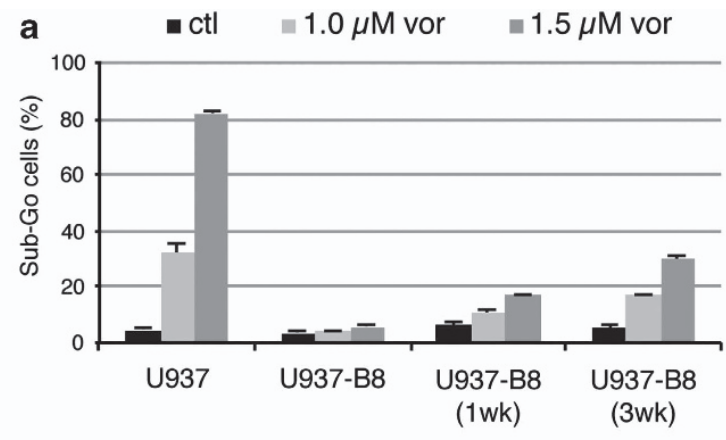

b

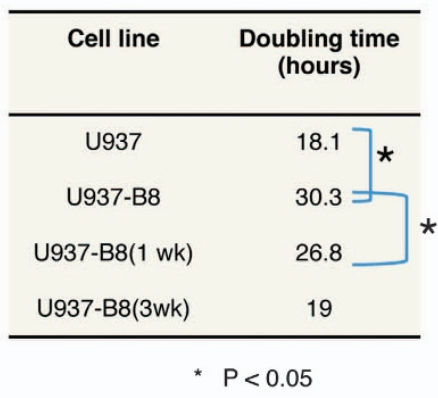

c

U937 $\rightarrow$ U937-B8 (1wk)

d $\because \mathrm{Ctl}=1.0 \mu \mathrm{M}$ Vor $\square 1.5 \mu \mathrm{M}$ Vor
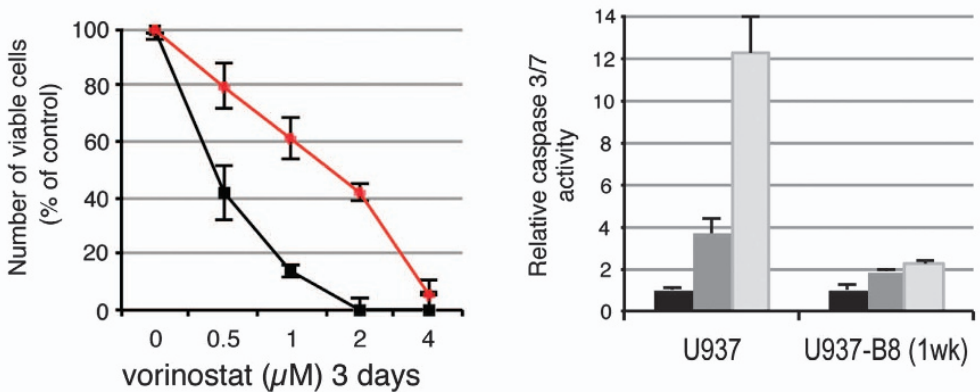

e

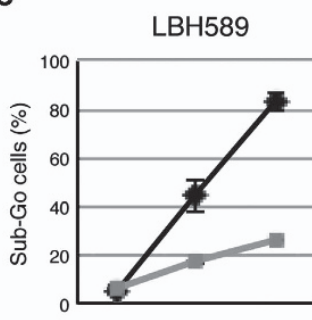

ctl $10 \mathrm{nM} 15 \mathrm{nM}$
Tubastatin

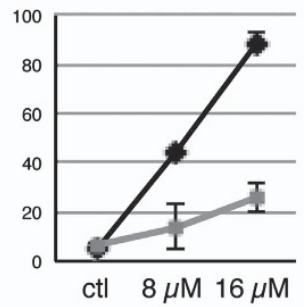

\section{MGCD0103}

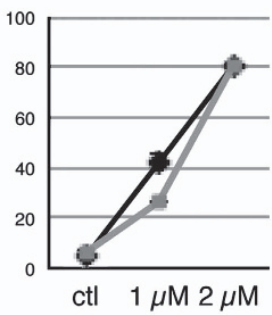

$\multimap$ U937

$=$ U937-B8(1wk) f $\quad$ U937 $\quad \mathrm{B} 8(1 \mathrm{wk}) \quad \mathrm{B} 8$

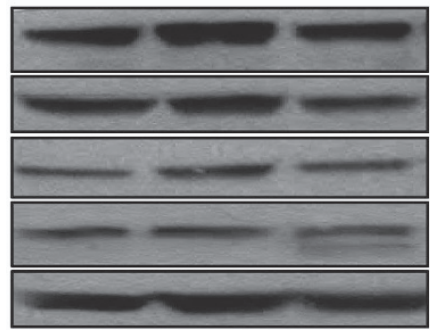

HDAC1
HDAC2
HDAC3
HDAC6
$\beta-A c t i n$

g

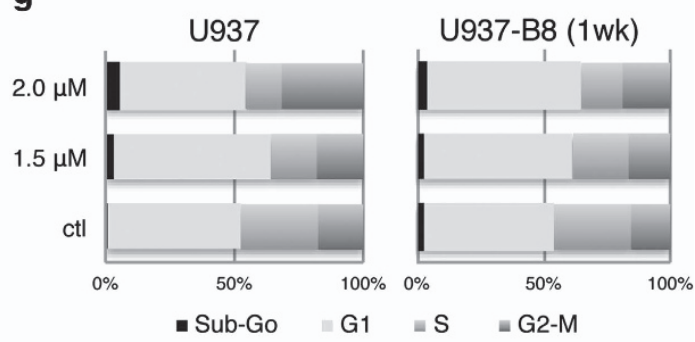

Figure 1 Chronic exposure to vorinostat induces resistance to pan-HDACi in the AML-related lymphoma cell line U937. U937-B8 cells were cultured in drug-free media for up to 3 weeks. After 2 days, 1 week and 3 weeks, U937-B8 cells' sensitivity to vorinostat was evaluated by quantification of cell death after $48 \mathrm{~h}$ exposure to the indicated concentration of vorinostat. Cell death was assessed by DNA fragmentation using PI staining and flow cytometry (a). Doubling time was measured by cell counting (b). U937B8 cells grown without vorinostat for 1 week and their parental cells U937 were treated with the indicated concentrations of vorinostat for $72 \mathrm{~h}$ before cell viability was evaluated by Titer-Glo assay (c) or $24 \mathrm{~h}$ before relative caspase 3 and 7 activation was evaluated by Caspase 3/7-Glo assay (d). Parental and resistant cells were exposed to the indicated concentrations of LBH589, tubastatin and MGCD0103 for $48 \mathrm{~h}$ and cell death was evaluated by DNA fragmentation using PI staining (e). U937 and U937-B8 cells grown in vorinostat and U937-B8 cells grown without vorinostat for 1 week were submitted to western blot analysis using whole-cell extract and the antibody indicated on the right (f). U937-B8 cells grown without vorinostat for 1 week and their parental cells U937 were treated with the indicated concentrations of vorinostat for $18 \mathrm{~h}$, and cell cycle analysis was performed by PI staining and flow cytometry $(\mathbf{g})$

protein light chain 3). When autophagy is induced, LC3 is cleaved and the smaller form LC3-II localizes to elongating autophagosome membranes and is subsequently degraded upon fusion of autophagosomes to lysosomes. CQ blocks degradation of autophagosomes thereby inducing LC3-II accumulation. In U937 cells and in U937-B8 cells, immunofluorescence shows that LC3 is diffuse in the cytoplasm at comparable levels (Figure $3 \mathrm{~b}$ ). However, after exposure to $25 \mu \mathrm{M} \mathrm{CQ}$, U937-B8 cells exhibit a considerable redistribution of LC3 from a diffuse cytoplasmic pattern to punctate 
structures, whereas only a modest accumulation of LC3 occurs in U937 cells, again illustrating increased basal autophagy in U937-B8 cells (Figure 3b). Like LC3, the chaperone p62 is continuously cleared in autophagosomes. By western blot, we further confirmed increased autophagy in U937-B8 cells by observing a greater accumulation of $p 62$ and LC3-II in response to blockage of autophagosome clearance by $\mathrm{CQ}$ (Figure 3c).

Beclin-1, a protein required for the initiation of autophagy, and atg7, which is necessary for the formation of autophagosomes, are both upregulated in U937-B8 cells (Figure 3d) compared with U937 cells. Further, upon activation of autophagy, atg5 is conjugated to atg12, and we observed increased atg5-atg12 conjugation in U937-B8 cells (Figure 3d). Lysosome-associated membrane glycoprotein-2 (Lamp-2) is a protein essential for the fusion of the autophagosome with the lysosome, ${ }^{21}$ and we observed that this protein is highly

Table $1 \mathrm{LD}_{50}$ of different therapies in parental and vorinostat-resistant U937 cells $(\mu \mathrm{M})$

\begin{tabular}{lcccc}
\hline Drug & $\begin{array}{c}\text { U937 } \\
\text { LD }_{\mathbf{5 0}}\end{array}$ & $\begin{array}{c}\text { U937-B8 } \\
\text { LD }_{\mathbf{5 0}}\end{array}$ & $\begin{array}{c}\text { U937-B8 } \\
\text { (1 week) } \text { LD }_{\mathbf{5 0}}\end{array}$ & $\begin{array}{c}\text { U937-B8 } \\
\text { (3 weeks) } \text { LD }_{\mathbf{5 0}}\end{array}$ \\
\hline Vorinostat & 1.1 & 9.8 & 4.7 & 4.9 \\
Chloroquine & 58.1 & 13.0 & 42.3 & 45.5 \\
Taxol & 0.0027 & 0.0025 & 0.0023 & 0.0022 \\
Cisplatin & 4.4 & 7.4 & 12.1 & 11.0 \\
Arsenic & 4.5 & 11.0 & 11.0 & 9.6 \\
trioxide & & \multirow{2}{*}{0.153} & 0.179 & 0.165 \\
Doxorubicin & 0.074 & 0.153 & & \\
\hline
\end{tabular}

upregulated in the U937-B8 cells. Notably, atg7 and Beclin-1 upregulation were also detected in the U937-E5 cell line (Supplementary Figure S3).

In U937 cells exposed to vorinostat, CQ induces accumulation of LC3-II similar to that observed in U937-B8 cells grown continuously in vorinostat (Figure 3b). We further confirmed that vorinostat treatment activates autophagy in U937 cells by western blot analysis showing degradation of p62 (Figure 3e).

The sensitivity of U937-B8 cells to CQ decreases following removal of vorinostat from the media for 1 week (Figure 2a); therefore, we tested whether the level of autophagy in U937-B8 cells correlated with the presence of vorinostat. We observed that autophagy decreases 1 week following the removal of vorinostat from the growth media, as shown by the reduced level of autophagosomes accumulating in response to $\mathrm{CQ}$ (Figure 4a). Additionally, we observed that washing off vorinostat from the media leads to the downregulation of atg7, Beclin-1, Lamp-2 and the atg5-atg12 conjugate (Figure 4b). Notably, this coincides with partial loss of resistance to vorinostat (Table 1), suggesting that increased autophagy correlates with the acquisition and maintenance of resistance to HDACi in U937-B8 cells. Importantly, this does not appear to be clone-specific, as increased sensitivity toward $C Q$ as well as increased levels of atg7 and Beclin-1 were also observed in U937-E5 cells (Supplementary Figures S3A and B).

To further show that the results are not limited to one cell line, we developed a vorinostat-resistant monoclonal cell line from the DLBCL line SUDHL6. The resistant SUDHL6-X cells are continuously grown in $4 \mu \mathrm{M}$ vorinostat. Consistent with a

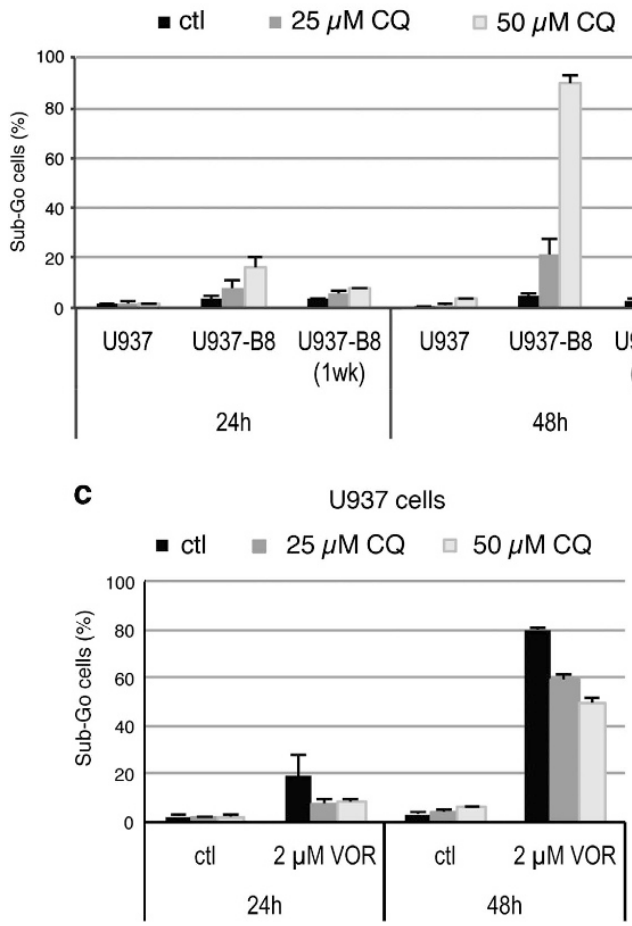

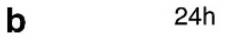

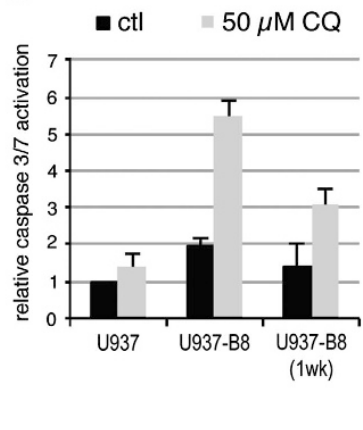

d U937 cells

$24 \mathrm{~h}$

- ctl $\square 50 \mu \mathrm{M} \mathrm{CQ}$

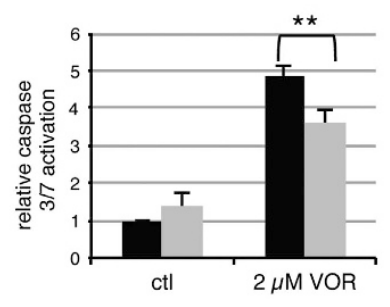

Figure 2 CQ overcomes resistance to vorinostat in U937-B8 cells but protects from vorinostat-induced toxicity in U937 cells. U937 and U937-B8 cells cultured in vorinostat and U937-B8 cultured 1 week in drug-free media were treated with or without the indicated concentrations of CQ for the indicated time before cell death (a) and relative caspase $3 / 7$ activity (b) were evaluated. U937 cells were treated with $2 \mu \mathrm{M}$ vorinostat with or without the indicated concentrations of CQ before cell death (c) and relative caspase $3 / 7$ activity (d) was evaluated 

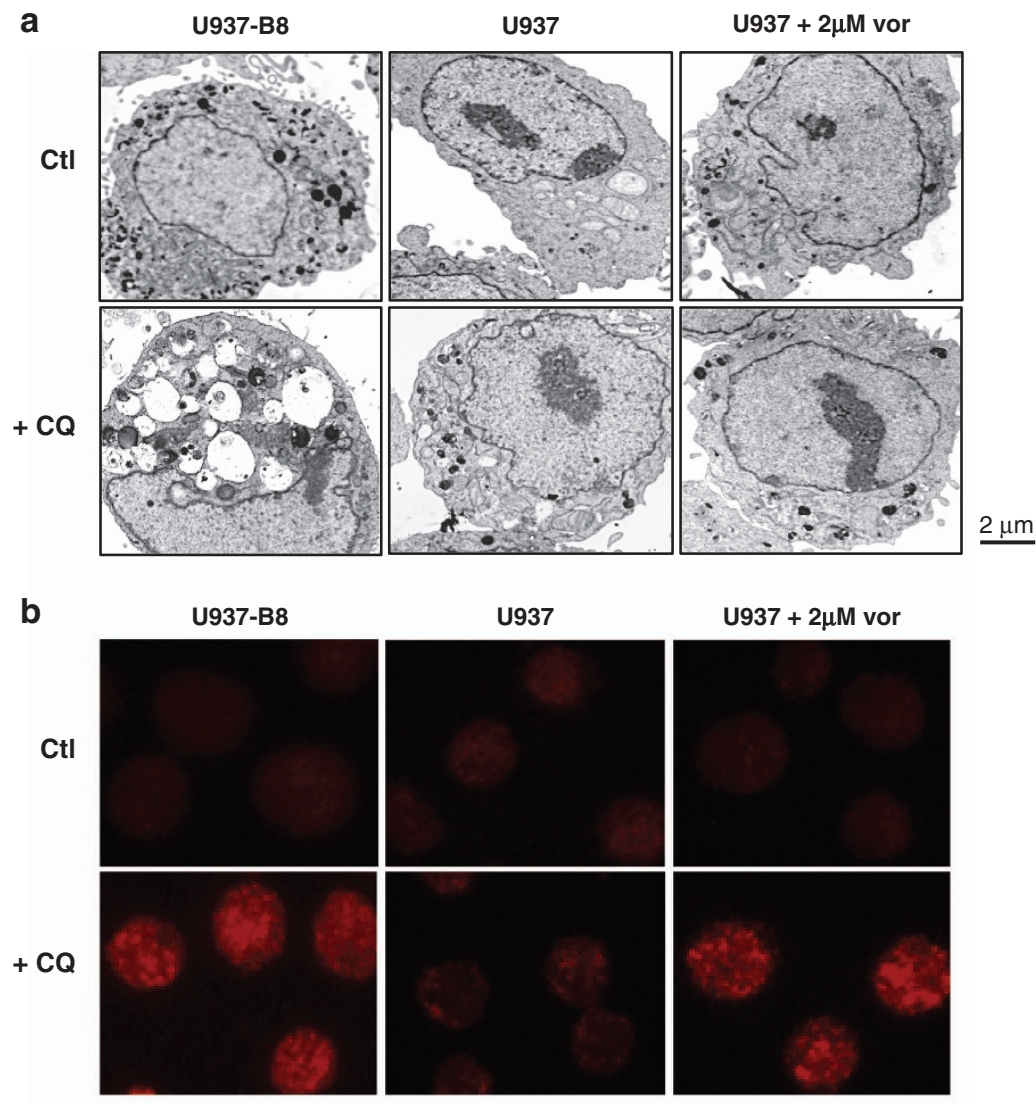

U937

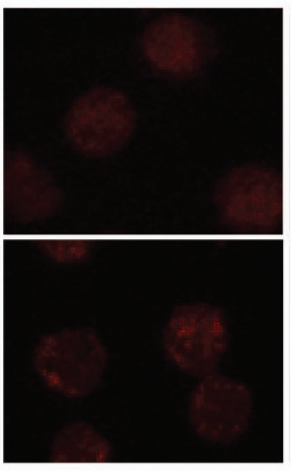

$\mathrm{U} 937+2 \mu \mathrm{M}$ vor

C

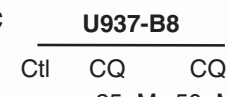

\begin{tabular}{ccc}
\multicolumn{3}{c}{ U937 } \\
\hline $\mathrm{ctl}$ & $\mathrm{CQ}$ & $\mathrm{CQ}$ \\
& $25 \mu \mathrm{M}$ & $50 \mu \mathrm{M}$
\end{tabular}

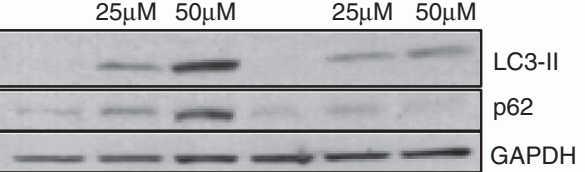

e

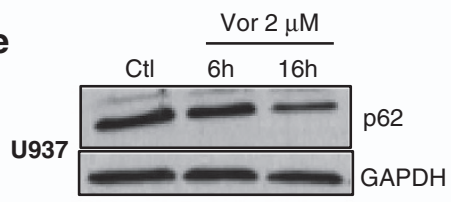

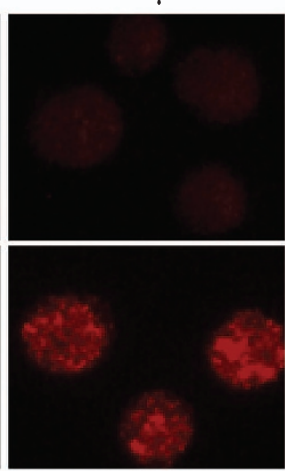

d

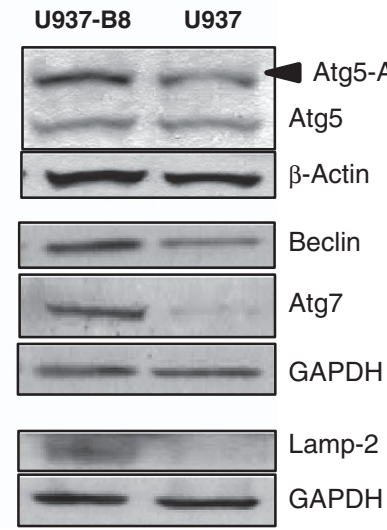

Figure 3 Short- and long-term exposure to vorinostat induces autophagy in U937 cells. U937-B8 cells cultured in $2 \mu \mathrm{M}$ vorinostat and their parental counterpart U937 (treated or not with $2 \mu \mathrm{M}$ vorinostat), each in the presence or absence of $25 \mu \mathrm{M} \mathrm{CQ}$ for $18 \mathrm{~h}$, were fixed and imaged by TEM (a) or analyzed by immunofluorescence microscopy using an antibody specific for LC3 (right panel) (b). U937-B8 cells cultured in $2 \mu \mathrm{M}$ vorinostat and parental U937 cells were treated or not with the indicated concentration of $\mathrm{CQ}$ for $18 \mathrm{~h}$ before total protein extracts were analyzed by western blotting using the antibodies indicated on the right (c and $\mathbf{d}$ ). The atg5 antibody detects the higher molecular weight conjugate with atg12. U937 cells treated with $2 \mu \mathrm{M}$ vorinostat for the indicated times followed by western blotting of total protein extracts with the antibodies indicated on the right (e)

what we observe in U937-B8 cells, resistance to vorinostat is slowly lost upon removal of the drug from the growth media (Supplementary Figure S4A). Moreover, SUDHL6-X cells are resistant to the induction of cell death by LBH589 and tubastatin but are sensitive to MGCD0103 (Supplementary Figure S4B). Importantly, $\mathrm{CQ}$ overcomes resistance to vorinostat in SUDHL6-X cells (Figure 5a), consistent with what we see in U937-B8 cells. However, at the same concentrations, $\mathrm{CQ}$ does not modulate vorinostat toxicity in the parental SUDHL6 cells (Figure 5b). We assessed autophagy by quantifying LC3-II accumulation following CQ exposure, using western blotting. In vorinostat-resistant SUDHL6-X cells, $50 \mu \mathrm{M} \mathrm{CQ}$ induces a substantial accumulation of cleaved LC3, as opposed to the parental cells, where LC3-II level does not increase (Figure 5c). Further, in parental cells treated with vorinostat, LC3-II does not significantly 
a

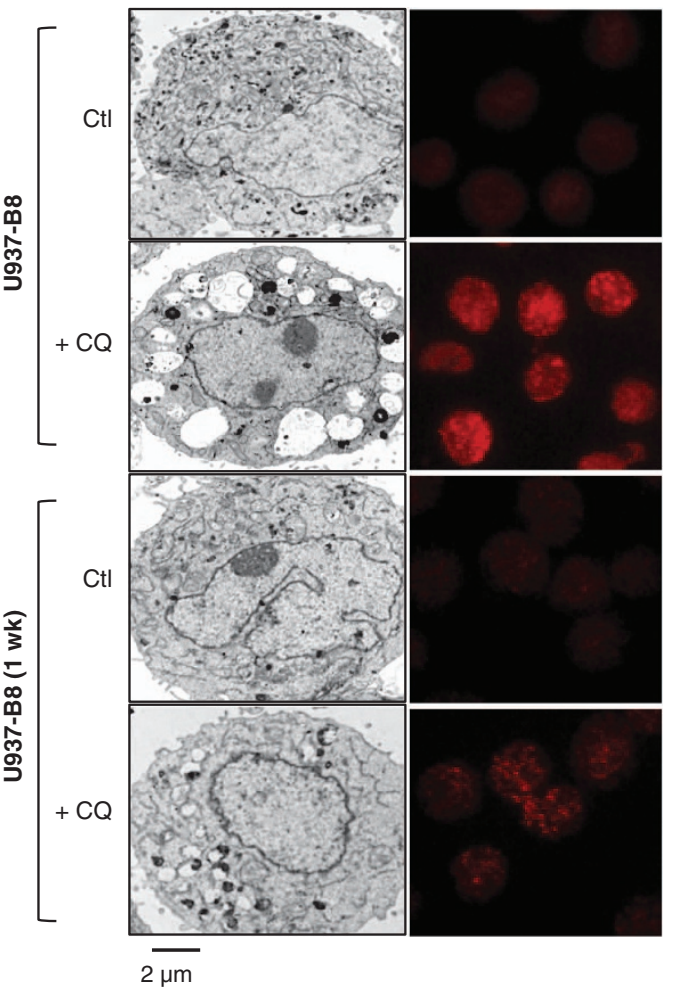

b

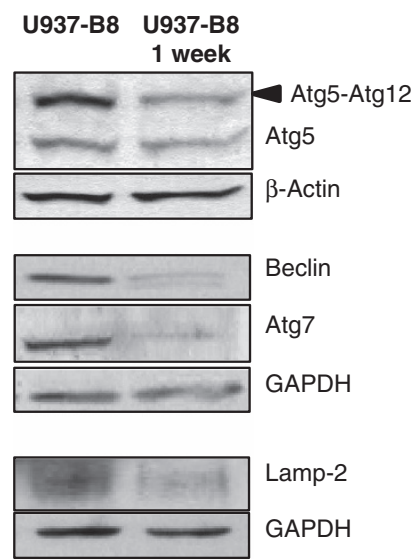

Figure 4 The presence of vorinostat correlates with increased autophagy in U937-B8 cells. U937-B8 cells cultured in $2 \mu \mathrm{M}$ vorinostat or 1 week in drug-free media, treated with or without $25 \mu \mathrm{M} \mathrm{CQ}$ for $18 \mathrm{~h}$, were fixed and imaged by TEM (left panel, scale bar $2 \mu \mathrm{m}$ ) or analyzed by immunofluorescence microscopy using antibody specific for LC3 (right panel) (a). U937-B8 cells cultured in $2 \mu \mathrm{M}$ vorinostat or 1 week in drug-free media analyzed by western blotting of total protein extracts with the antibodies indicated on the right $(\mathbf{b})$

accumulate in the presence of $\mathrm{CQ}$, indicating that autophagy is not induced by de novo vorinostat treatment. Unlike U937B8 cells, SUDHL6-X cells do not significantly show elevated protein levels of Beclin-1, atg7 or atg5-atg12 conjugates, as measured by western blotting (Figure $5 \mathrm{~d}$ ). In contrast, Lamp-2 protein is highly upregulated, consistent with our observation in U937-B8 cells (Figure 3d). Overall, the results obtained in these vorinostat-resistant DLBCL cells support a prosurvival role of autophagy induced during acquisition of resistance to vorinostat. However, apoptosis of parental cells exposed to vorinostat is not affected by inhibition of autophagy in this cellular model.

To confirm that autophagy has a proapoptotic role in U937 cells, we reasoned that blocking autophagy by genetically silencing key proteins in the autophagic process should protect cells from vorinostat-induced cell death, similar to chemically inhibiting autophagy with CQ. To this end, we disrupted autophagy by knocking down Beclin-1. Consistent with our hypothesis, we found that following downregulation of Beclin-1 by short hairpin RNA (shRNA), vorinostat toxicity in U937 cells was decreased (Figure 6a, left panel). We also downregulated Lamp-2 and indeed found that autophagy was suppressed, as observed by the accumulation of p62, and that U937 cells were protected from vorinostat-induced toxicity (Figure 6a, right panel). In contrast, even a modest knockdown of Lamp-2 or Beclin-1 induces cell death in U937-B8 cells in the presence of $2 \mu \mathrm{M}$ vorinostat (Figure $6 \mathrm{~b}$ ), confirming that autophagy is required for survival of the resistant cells.

As autophagy contributes to vorinostat-induced apoptosis in U937 cells, we reasoned that an inducer of autophagy would synergize with vorinostat to induce apoptosis in U937 cells. mTOR (mammalian target of rapamycin) prevents initiation of autophagy by phosphorylating unc-51-like kinase 1,22 and rapamycin induces autophagy by relieving this negative regulation. Indeed, we observe that $50 \mathrm{nM}$ rapamycin inhibits the mTOR pathway, as evidenced by dephosphorylation of its downstream target 4EBP1, and induces autophagy in $U 937$ cells, as reflected by p62 degradation revealed by western blotting (Figure 7b). At this concentration, rapamycin alone does not exhibit toxicity but increases vorinostat-induced cell death (Figure 7a). Rapamycin also increases cell death induced by the HDACi LBH589 (Supplementary Figure S5). mTOR exists in two functionally distinct complexes, mTORC1 and mTORC2. Whereas rapamycin inhibits only mTORC1 functions, BEZ235 was developed to inhibit both complexes and the upstream phosphatidylinositol 3-kinases. ${ }^{23-25}$ Similar to rapamycin, cotreatment of U937 cells with BEZ235 increases cell death induced by vorinostat (Figure 7a). BEZ235 inhibits mTOR pathway in U937 cells as seen by western blotting showing dephosphorylation of 4EBP1, and also induces autophagy as shown by p62 degradation (Figure 7b). 

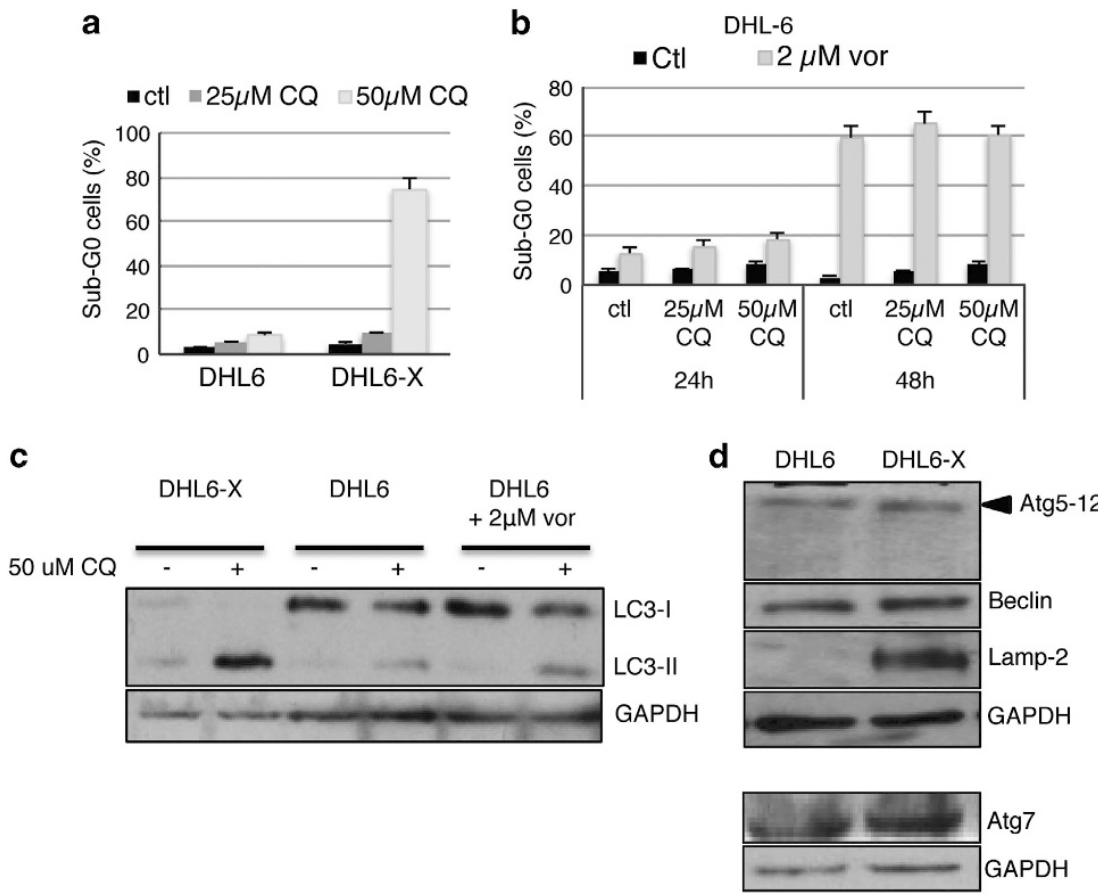

Figure 5 Acquired resistance to vorinostat in SUDHL6 cells correlates with increased autophagy and is overcome by CQ. SUDHL6-X and their parental counterpart SUDHL6 cells were treated with the indicated concentration of vorinostat for $48 \mathrm{~h}$ before cell death was assessed by PI staining with flow cytometry (a). SUDHL6 cells were treated with $2 \mu \mathrm{M}$ vorinostat with or without the indicated concentrations of CQ before cell death was evaluated (b). SUDHL6-X cells cultured in $2 \mu \mathrm{M}$ vorinostat and parental SUDHL6 cells exposed or not to $2 \mu \mathrm{M}$ vorinostat were treated or not with the indicated concentration of $\mathrm{CQ}$ for $6 \mathrm{~h}$ before total protein extracts were analyzed by western blotting using the antibodies indicated on the right (c). Western blot analysis performed with total extract of SUDHL6 and SUDHL6-X cells using the antibodies indicated on the right (d)

Despite the fact that U937-B8 cells display constitutively elevated autophagy when cultured in vorinostat, we find that the activity of the mTOR pathway is not significantly changed. Indeed, western blotting shows phosphorylated 4EBP1 and mTOR unchanged in U937-B8 cells (Figure 7c). In the resistant cells, rapamycin and BEZ235 do suppress mTOR signaling and augment autophagy (Figures $7 \mathrm{~d}$ and e, bottom panels). However, U937-B8 cells are insensitive to induction of cell death by rapamycin and BEZ235, even when they are exposed to higher concentrations of vorinostat (Figures $7 d$ and e, upper panels).

\section{Discussion}

HDACi are known to restore the expression of silenced tumor suppressor genes in cancer cells through their ability to induce histone acetylation. Still, many studies have shown that HDACi have multiple mechanisms of action besides modulation of gene expression. ${ }^{26}$ Patients with a variety of hematological malignancies have shown complete remissions in clinical trials with $\mathrm{HDACi} ;{ }^{27}$ however, the majority of patients derive no benefit from HDACi monotherapy. Many patients exhibit de novo resistance and others acquire resistance during long-term exposure. Biomarkers for identification of patients likely to respond to $\mathrm{HDACi}$, as well as successful combination strategies, are therefore needed. Here, in an attempt to better understand the key molecular effectors of resistance to vorinostat, we developed in vitro models of resistance to vorinostat using the monocytic-like lymphoma cell line U937 and the DLBCL SUDHL6 line.
Strikingly, vorinostat-resistant cells die in response to the antimalarial drug $\mathrm{CQ}$, whereas their parental counterparts do not. $\mathrm{CQ}$ and its derivatives have been shown to inhibit autophagy by blocking autophagosomal function. ${ }^{28}$ Although the function of autophagy in tumor biology remains elusive, it is clear that the tumor microenvironment, chemotherapy and stress can tip the scale in favor of a prosurvival or proapoptotic role of autophagy. Our model system has been exploited to understand the role of autophagy in response and resistance to vorinostat. By understanding the duality of autophagy in cell survival and cell death, we hope to augment the therapeutic potential of HDACi's by appropriately modifying the autophagic pathway.

Accumulating evidence demonstrate that autophagy is involved both in cancer development and in adaptation of cancer cells to chemotherapy. ${ }^{29,30}$ Thus, inhibition of autophagy is emerging as a novel cancer strategy, now in early stages of clinical trials as either monotherapy or in combination with other agents (http://www.cancer.gov/clinicaltrials). However, studies are inconsistent, and autophagy has been linked to opposing functions, survival or cell death, depending on the model or stimulus used. ${ }^{18}$ Studies showing that induction of autophagy by HDACi can occur along with cell death suggest that autophagy promotes cell death. ${ }^{31-33}$ In this study we clearly demonstrate, by knockdown of the essential autophagy genes Beclin-1 and Lamp-2, that killing of U937 cells by de novo HDACi treatment is enhanced by the activation of autophagy.

Conversely, several studies have shown that HDACi can induce autophagy as a prosurvival mechanism. Notably, in 
a
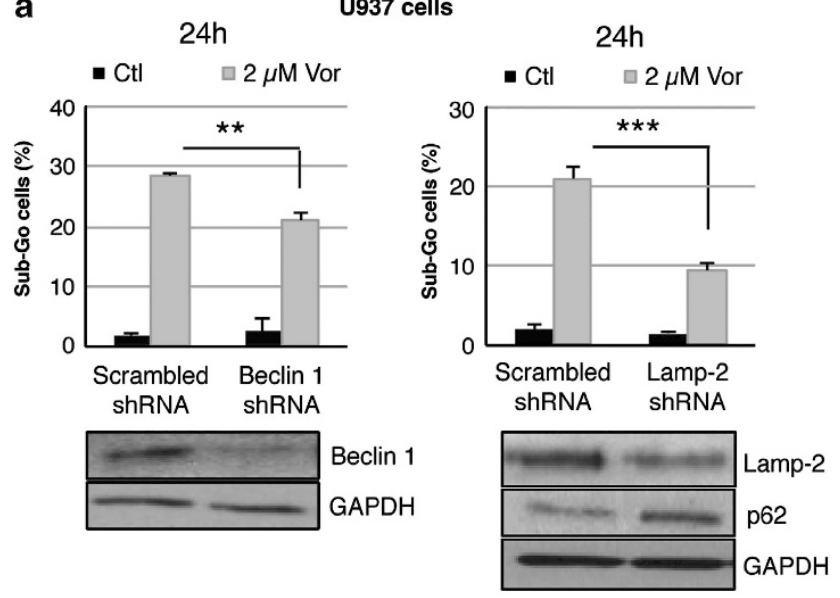

b

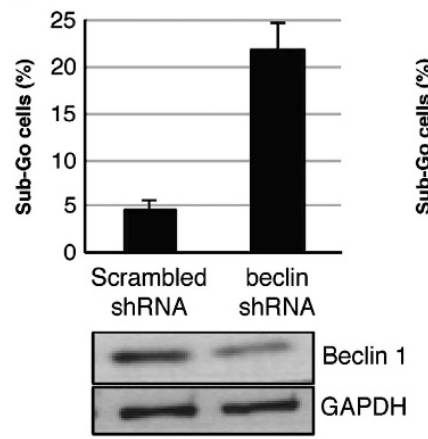

U937-B8 cells

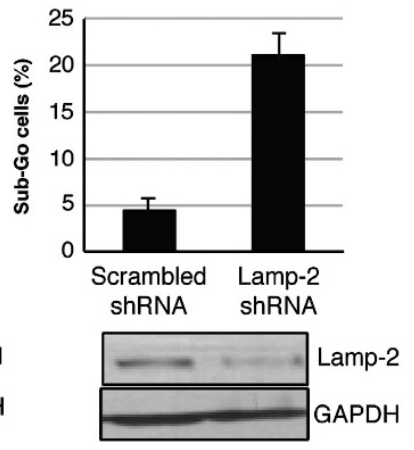

Figure 6 Autophagy drives acquired resistance to vorinostat in U937-B8 cells but contributes to vorinostat-induced toxicity in their parental cells. U937 cells were infected with Scrambled (non-targeting) or Beclin-1 or Lamp-2 shRNA. $48 \mathrm{~h}$ after infection, cells were treated with or without $2 \mu \mathrm{M}$ vorinostat for $24 \mathrm{~h}$, followed by quantification of cell death by PI staining (upper panels). $72 \mathrm{~h}$ after the infection, cells were harvested for western blotting of total protein extracts with the antibodies indicated (bottom panel) (a). U937-B8 cells were infected with Scrambled or Beclin1 or Lamp-2 shRNAs. Immediately after infection, $2 \mu \mathrm{M}$ vorinostat was added to the media. After $48 \mathrm{~h}$, cells were harvested for western blotting of total protein extracts with the antibodies indicated (bottom panels). After $72 \mathrm{~h}$, cell death was quantified by PI staining (top panel) (b)

malignant peripheral nerve sheath tumors cells, inhibition of HDACi-induced autophagy enhances HDACi proapoptotic effects, in both HDACi-resistant and -sensitive cells. ${ }^{34}$ Additional studies in colon cancer cells and chronic myelogenous leukemia cells reported that genetic or chemical inhibition of autophagy increased HDACi toxicity. ${ }^{35,36}$ Some mechanisms of how autophagy enables the cell to survive in unfavorable conditions have been suggested, for example, by the removal of apoptotic effectors (caspase 8$)^{37}$ and by the degradation of misfolded/aggregated proteins ${ }^{18}$ and damaged organelles, which restores metabolic homeostasis by recycling metabolites and prevents ROS accumulation. ${ }^{38}$

In this study, we observed that U937-B8 cells have a significantly higher basal level of ubiquitylated proteins (Supplementary Figure S6), indicative of an accumulation of misfolded proteins. Furthermore, $C Q$ induces a substantial further accumulation of ubiquitylated proteins in U937-B8 cells cultured in vorinostat, but not in U937 cells with or without vorinostat. Thus, we hypothesize that autophagy mediates its cytoprotective effect in vorinostat-resistant cells by clearing accumulated misfolded/aggregated proteins. HDAC6 is responsible for the acetylation of tubulin and HSP90, and it has a critical role in the cellular response to the accumulation of misfolded proteins. ${ }^{39}$ Of note, vorinostat-resistant cells are still sensitive to MGCD0103, which does not significantly inhibit HDAC6 as opposed to other HDACi, tested (LBH589 and tubastatin). These data evoke the possibility that inhibition of HDAC6 and altered processing of misfolded protein have a role in vorinostat toxicity. This is in keeping with a study by Fiskus et al. $^{13}$ describing the loss of HDAC6, hyperacetylation of hsp90 and increased sensitivity to the hsp90 inhibitor 17-AAG in LAQ824-resistant AML cell line. ${ }^{13}$ Consistent with our model, they reported cross-resistance to other pan-HDACi vorinostat and LBH589.

mTOR has a central role as a regulator of cell death induced by overactivation or prolonged activation of autophagy. When mTOR is inhibited during mild cellular stress, a critical feedback reactivates mTOR through the phosphorylation of Akt and terminates autophagy. ${ }^{40}$ Gupta et al. ${ }^{41}$ demonstrated that LBH589 was able to inhibit Akt phosphorylation upon mTOR inhibition by rapamycin, thus impeding reactivation of mTOR. Further, they showed that the combination of rapamycin and LBH led to synergistic killing of lymphoma cells, ${ }^{41}$ as we observe in U937 cells. Our results showing unchanged mTOR status in U937-B8 cells suggest that autophagy may be induced independently of mTOR signaling during acquisition of resistance to vorinostat. We propose a model (Figure 7f) in which de novo exposure to vorinostat induces autophagy, which reduces the cell viability. Hence, initial vorinostat treatment may be combined with inducers of autophagy, for instance mTOR inhibitors, to enhance the efficacy of therapy. However, as cells acquire resistance to vorinostat during the course of long-term exposure, autophagy becomes essential for cells to survive vorinostat toxicity, and therefore, cells gain sensitivity to inhibitors of autophagy.

The potential role of autophagy in the therapeutic response to anticancer agents has been controversial, as opposing results are obtained from different cell lines and/or chemotherapy. For the first time, we show that exposure to the same drug in the same cell model can induce autophagy with opposing outcomes. Furthermore, we provide a model to dissect autophagy and to study how it adapts from a weakness to a weapon helping cancer cells survive therapeutic stress.

\section{Materials and Methods}

Reagents. Vorinostat was obtained from Merck (Boston, MA, USA), LBH589 from Novartis (Dorval, QC, Canada), MGCD0103 from Methylgene Princeton, NJ, USA) and tubastatin was purchased from Biovision (Milpitas, CA, USA). All drugs were resuspended in DMSO.

Cell lines. U937 cells were obtained from ATCC (Manassas, VA, USA). SUDHL6 cells were kindly provided by Dr. Ari Melnick (Weill Cornell Medical College, Manhattan, NY, USA). Cells were maintained in RPMI supplemented with $10 \%$ fetal bovine serum (FBS) at $37^{\circ} \mathrm{C}$ with $5 \% \mathrm{CO}_{2}$.

Vorinostat-resistant cell lines. The same protocol was followed for U937 and SUDHL6 cells. Cells were seeded at $0.3 \times 10^{6}$ cells per $\mathrm{ml}$ and maintained in $0.5 \mu \mathrm{M}$ vorinostat until cells started growing and reached confluency. Cells were then plated at $0.3 \times 10^{6}$ cells per $\mathrm{ml}$ and exposed to $1 \mu \mathrm{M}$ concentration of vorinostat. That protocol was repeated until the vorinostat concentration reached 

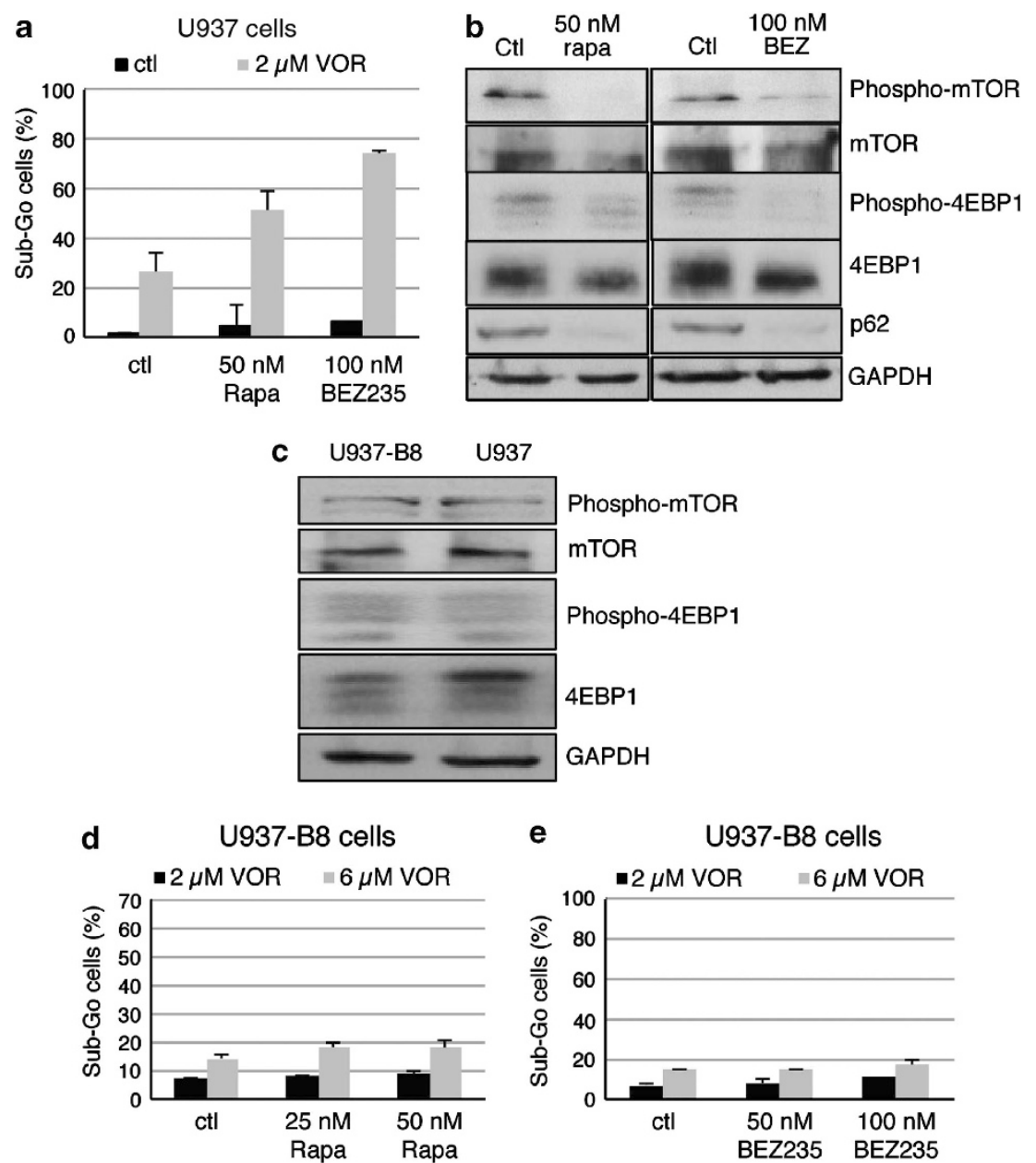

e

U937-B8 cells
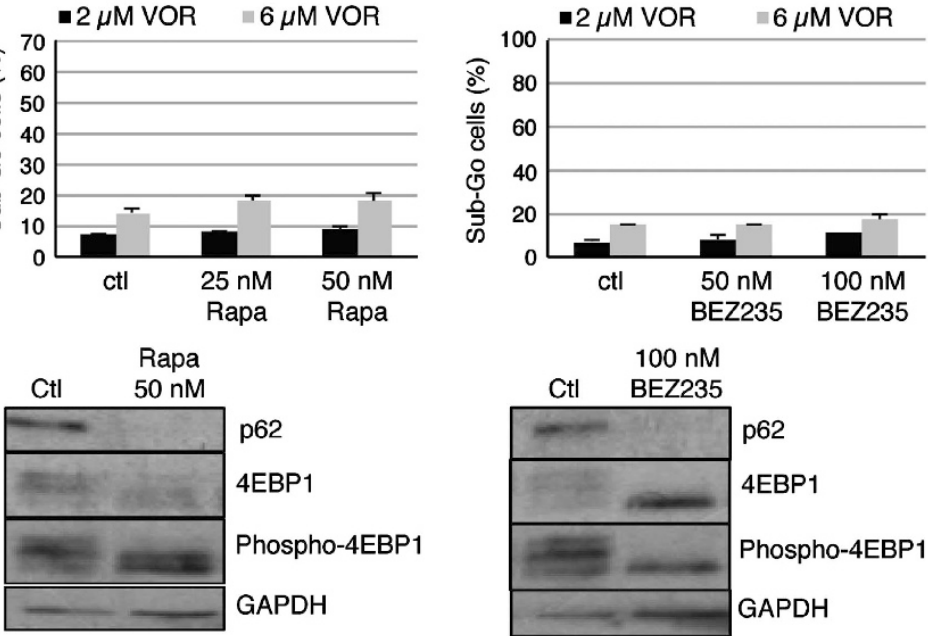

$100 \mathrm{nM}$
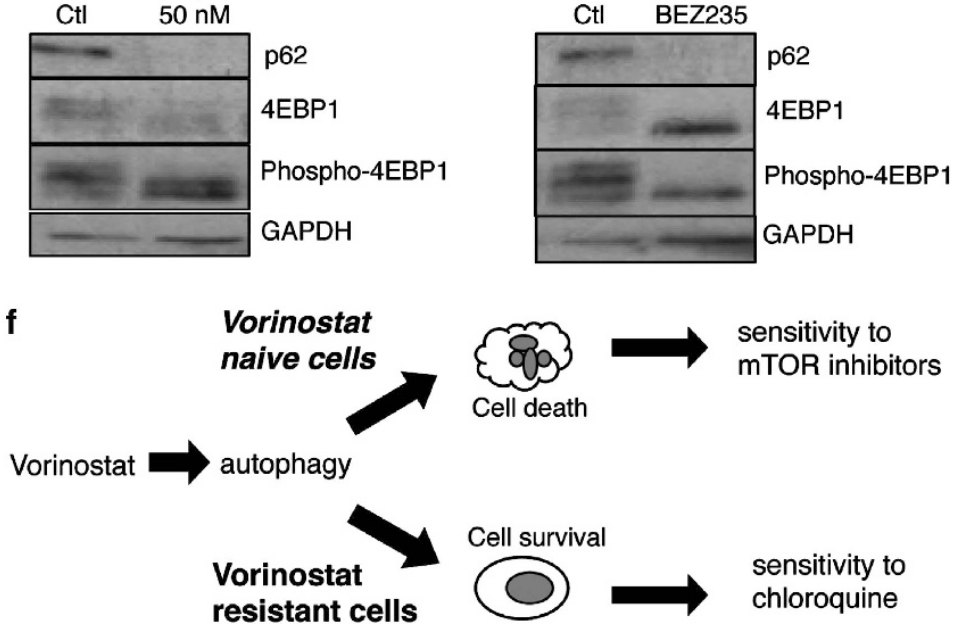

Figure 7 mTOR inhibitors induce autophagy and increase HDACi-induced cell death in U937 cells. U937 cells treated with the indicated concentrations of rapamycin or BEZ235 for $24 \mathrm{~h}$ before cell death was evaluated by PI staining (a). U937 cells treated for $18 \mathrm{~h}$ with rapamycin or BEZ235 were analyzed by western blotting of total extract with the antibodies indicated on the right (b). U937-B8 and U937 cells were analyzed by western blotting of total extract using the indicated antibodies (c). U937-B8 cells treated with the indicated concentration of rapamycin (lower panel in d) or BEZ235 (lower panel in e) for $18 \mathrm{~h}$ were analyzed by western blotting with the antibodies indicated. U937-B8 cells cultured in 2 and $6 \mu \mathrm{M}$ vorinostat were treated with or without rapamycin (upper panel in d) or BEZ235 (upper panel in e) for $24 \mathrm{~h}$ before cell death was quantified by PI staining. Our model: in naive cells, vorinostat induces autophagy, which then triggers cell death. Hence, inducers of autophagy may be used in combination with vorinostat, to enhance the induction of apoptosis. Cells maintained in vorinostat long term develop resistance to induction of cell death but still activate autophagy. In this setting, resistant cells rely on autophagy to subsist vorinostat. Resistant cells are therefore vulnerable to inhibitors of autophagy (f) 
$2 \mu \mathrm{M}$. A polyclonal population was obtained and separated into monoclonal populations by limiting dilution in 96-well/plates in the presence of 2 and $4 \mu \mathrm{M}$ vorinostat, respectively. All resistant cell lines were maintained in RPMI supplemented with $10 \% \mathrm{FBS}$ at $37^{\circ} \mathrm{C}$ with $5 \% \mathrm{CO}_{2}$ with $2 \mu \mathrm{M}$ vorinostat for U937 clones or $4 \mu \mathrm{M}$ for SUDHL6-resistant cells.

PI staining. Cells were seeded at $2 \times 10^{5}$ cells per $\mathrm{ml}$ and treated as described. Cells were washed in buffer (PBS/5\% FBS/0.01 M NaN ${ }_{3}$ ) at $4{ }^{\circ} \mathrm{C}$, pelleted and resuspended in $0.5 \mathrm{ml}$ of hypotonic fluorochrome solution containing $50 \mu \mathrm{g} / \mathrm{ml} \mathrm{PI}$ (BD Pharmingen, San Diego, CA, USA), $0.1 \mathrm{M}$ sodium citrate and $0.1 \%$ Triton X-100. Fluorescence was detected using a FACS Calibur flow cytometer, and analysis was carried out using the CellQuest software (Becton Dickinson, San Jose, CA, USA). A total of 7000 events were collected per sample. Cells undergoing DNA fragmentation and apoptosis were defined as events with $\mathrm{PI}$ fluorescence weaker than the G0-G1 cell cycle peak (sub-G0).

Transmission electron microscopy. Samples for electron microscopy were fixed in $2.5 \%$ glutaraldehyde $0.1 \mathrm{M}$ sodium cacodylate buffer, $\mathrm{pH} 7.4$, overnight at $4{ }^{\circ} \mathrm{C}$ and processed for flat embedding in Epon 812 at the Facility for Electron Microscopy Research at McGill University (Montreal, Quebec, Canada). Sections were examined with an FEI Tecnai $\mathrm{G}^{2}$ Spirit $120 \mathrm{kV}$ TEM and Gatan Ultrascan $10002 k \times 2 k$ CCD Camera System Model 895 (FEl Company, Hillsboro, Oregon USA).

Caspase 3/7 assay. Caspase 3/7 activity was measured with the CaspaseGlo 3/7 assay according to the instructions from the manufacturer (Promega, Madison, WI, USA).

Western blotting. Whole-cell extracts were prepared in lysis buffer $(1 \%$ Triton X-100, $150 \mathrm{mM} \mathrm{NaCl}, 20 \mathrm{mM}$ Tris (pH 8.0)), run on SDS-polyacrylamide gels and transferred onto nitrocellulose membranes. Membranes were blocked and subsequently probed with antibodies against HDAC3, HDAC6, Lamp-2, atg7, p62, Beclin-1 (Santa Cruz Biotechnology, Santa Cruz, CA, USA), phosphorylated and total 4EBP1, phosphorylated and total mTOR (Cell Signalling, Danvers, MA, USA) HDAC1, HDAC2 (Millipore, Billerica, MA, USA), atg5, LC3, GAPDH (Cell Signaling) and $\beta$-actin (Sigma, Oakville, ON, Canada). Membranes were then probed with anti-rabbit or anti-mouse antibody (Amersham; GE Healthcare, Baie d'Urfé, QC, Canada) or anti-goat (Santa Cruz Biotechnology) secondary antibodies, followed by visualization with enhanced chemiluminescence (ECL; Amersham)

Short hairpin RNA. shRNA deoxyoligonucleotide sequences were designed as follows: Lamp-2, 5'-GATCCCCGTCTTATGCATTGGAACTTTTCAAGAGAAA GTTCCAATGCAT AAGACTTTTTC-3' and 5'-TCGAGAAAAAGTCTTATGCATTGGAACTTTCTCTTGAAAAG TTCCAATGCATAAGACGGG-3'; scrambled, 5' -GATC CCCGGTGAAGTACCGCTAAG GATTCAAGAGATCCTTAGCGGTACTTCACCTT TTTTC- $3^{\prime}$ and 5'-TCGAGAAAAAGGT GAAGTACCGCTAAGGATCTCTTGAATC CTTAGCGGTACTTCACCGGG-3'; and Beclin-1, 5'-GATCCCCCAGTTTGGCACA ATCAATATTCAAGAGATATTGATTGTGCCAAACTGT TTTTC-3' and 5'-TCGAGA AAAACAGTTTGGCACAATCAATATCTCTTGAATATTGATTGTGCCAAACTGGGG$3^{\prime}$. shRNA sequences were hybridized and cloned into pSuperRetro vector digested with Xhol and Hindll.

Downregulation of Lamp-2 and Beclin-1. Vectors were packaged in 293T phoenix amphotropic cells using JetPrime transfection reagent (Polyplus transfection, New York, NY, USA). 48 and $72 \mathrm{~h}$ following transfection, viral supernatants were collected and pooled. Viral particles were concentrated 10 -fold by precipitation in $8.5 \%$ polyethylene glycol, $0.4 \mathrm{M} \mathrm{NaCl}$ overnight at $4^{\circ} \mathrm{C}$ with centrifugation at 3000 r.p.m. for $30 \mathrm{~min}$. Concentrated viral particles were resuspended in RPMI 10\% FBS containing $4 \mu \mathrm{g} / \mathrm{ml}$ polybrene. U937 and U937-B8 cells were incubated with viral particles at $37{ }^{\circ} \mathrm{C}$ with $5 \% \mathrm{CO}_{2}$ for $24 \mathrm{~h}$ at a starting confluency of $4 \times 10^{5}$ cells per ml.

Immunofluorescence. A total of 30000 cells were cytospun onto glass slides at 500 r.p.m. for $5 \mathrm{~min}$. Cells were fixed in methanol, permeabilized in PBS $0.1 \%$ with Triton TX-100 for 30 min and then blocked with PBS $0.5 \%$ BSA for $1 \mathrm{~h}$. Cells were incubated with antibody against LC3 or ubiquitin (Cell Signaling) for $2 \mathrm{~h}$, washed three times for $10 \mathrm{~min}$ in PBS $0.1 \%$ with Triton TX-100 and then incubated with secondary anti-rabbit antibody coupled to AlexaFluor488 or AlexaFluor594
(Invitrogen from Life Technologies, Grand Island, NY, USA) for $1 \mathrm{~h}$. Finally, cells were washed three times in PBS with $0.1 \%$ Triton TX-100 for 10 min, mounted in $90 \%$ glycerol and visualized with a fluorescent microscope (Leica, Concord, ON, Canada).

Statistics. All experiments were repeated at least three times. Significance was determined by Student's t-test or ANOVA using Prism version 4.0 (GraphPad, La Jolla, CA, USA).

\section{Conflict of Interest}

The authors declare no conflict of interest.

Acknowledgements. This research was supported by CIHR Grant No. MOP12863. We thank Merck for providing vorinostat and financial support. LBH589 was kindly provided by Novartis. We also thank Ryan E Henry for providing transduction protocols for knockdown experiments.

\section{Author contributions}

D Dupéré-Richer designed and performed research, analyzed data and wrote the paper. M Kinal and TH Nielsen performed some of the experiments and helped with preparation of the manuscript. WH Miller, S del Rincon and F Pettersson helped with experimental design, data analysis and interpretation, and preparation of the manuscript.

1. Olsen EA, Kim YH, Kuzel TM, Pacheco TR, Foss FM, Parker S et al. Phase llb multicenter trial of vorinostat in patients with persistent, progressive, or treatment refractory cutaneous T-cell lymphoma. J Clin Oncol 2007; 25: 3109-3115.

2. Chiba T, Yokosuka O, Fukai K, Kojima H, Tada M, Arai M et al. Cell growth inhibition and gene expression induced by the histone deacetylase inhibitor, trichostatin $\mathrm{A}$, on human hepatoma cells. Oncology 2004; 66: 481-491.

3. Choudhary C, Kumar C, Gnad F, Nielsen ML, Rehman M, Walther TC et al. Lysine acetylation targets protein complexes and co-regulates major cellular functions. Science 2009; 325: 834-840.

4. Petruccelli LA, Dupere-Richer D, Pettersson F, Retrouvey H, Skoulikas S, Miller Jr WH. Vorinostat induces reactive oxygen species and DNA damage in acute myeloid leukemia cells. PLoS One 2011; 6: e20987.

5. Ruefli AA, Ausserlechner MJ, Bernhard D, Sutton VR, Tainton KM, Kofler $R$ et al. The histone deacetylase inhibitor and chemotherapeutic agent suberoylanilide hydroxamic acid (SAHA) induces a cell-death pathway characterized by cleavage of Bid and production of reactive oxygen species. Proc Natl Acad Sci USA 2001; 98: 10833-10838.

6. Vrana JA, Decker RH, Johnson CR, Wang Z, Jarvis WD, Richon VM et al. Induction of apoptosis in U937 human leukemia cells by suberoylanilide hydroxamic acid (SAHA) proceeds through pathways that are regulated by $\mathrm{Bcl}-2 / \mathrm{Bcl}-\mathrm{XL}$, c-Jun, and p21CIP1, but independent of p53. Oncogene 1999; 18: 7016-7025.

7. Singh TR, Shankar S, Srivastava RK. HDAC inhibitors enhance the apoptosis-inducing potential of TRAIL in breast carcinoma. Oncogene 2005; 24: 4609-4623.

8. Garcia-Manero G, Yang H, Bueso-Ramos C, Ferrajoli A, Cortes J, Wierda WG et al. Phase 1 study of the histone deacetylase inhibitor vorinostat (suberoylanilide hydroxamic acid [SAHA]) in patients with advanced leukemias and myelodysplastic syndromes. Blood 2008; 111: 1060-1066.

9. Kirschbaum MH, Goldman BH, Zain JM, Cook JR, Rimsza LM, Forman SJ et al. A phase 2 study of vorinostat for treatment of relapsed or refractory Hodgkin lymphoma: Southwest Oncology Group Study S0517. Leuk Lymphoma 2011; 53: 259-262.

10. Fantin VR, Richon VM. Mechanisms of resistance to histone deacetylase inhibitors and their therapeutic implications. Clin Cancer Res 2007; 13: 7237-7242.

11. Hu Y, Lu W, Chen G, Zhang H, Jia Y, Wei Y et al. Overcoming resistance to histone deacetylase inhibitors in human leukemia with the redox modulating compound betaphenylethyl isothiocyanate. Blood 2010; 116: 2732-2741.

12. Bandyopadhyay D, Mishra A, Medrano EE. Overexpression of histone deacetylase 1 confers resistance to sodium butyrate-mediated apoptosis in melanoma cells through a p53-mediated pathway. Cancer Res 2004; 64: 7706-7710.

13. Fiskus W, Rao R, Fernandez P, Herger B, Yang Y, Chen J et al. Molecular and biologic characterization and drug sensitivity of pan-histone deacetylase inhibitor-resistant acute myeloid leukemia cells. Blood 2008; 112: 2896-2905

14. Dai $Y$, Rahmani M, Dent $P$, Grant $S$. Blockade of histone deacetylase inhibitor-induced RelA/p65 acetylation and NF-kappaB activation potentiates apoptosis in leukemia cells through a process mediated by oxidative damage, XIAP downregulation, and c-Jun $\mathrm{N}$-terminal kinase 1 activation. Mol Cell Biol 2005; 25: 5429-5444

15. Mitsiades N, Mitsiades CS, Richardson PG, McMullan C, Poulaki V, Fanourakis G et al. Molecular sequelae of histone deacetylase inhibition in human malignant $B$ cells. Blood 2003; 101: 4055-4062 
16. Carew JS, Medina EC, Esquivel JA 2nd, Mahalingam D, Swords R, Kelly $K$ et al. Autophagy inhibition enhances vorinostat-induced apoptosis via ubiquitinated protein accumulation. J Cell Mol Med 2010; 14: 2448-2459.

17. Wirawan E, Berghe TV, Lippens S, Agostinis P, Vandenabeele P. Autophagy: for better or for worse. Cell Res 2011

18. Wirawan E, Vanden Berghe T, Lippens S, Agostinis P, Vandenabeele P. Autophagy: for better or for worse. Cell Res 2012; 22: 43-61.

19. Petruccelli LA, Dupere-Richer D, Pettersson F, Retrouvey H, Skoulikas S, Miller Jr WH. Vorinostat induces reactive oxygen species and DNA damage in acute myeloid leukemia cells. PLoS One 2011; 6: e20987.

20. Ohkuma S, Poole B. Fluorescence probe measurement of the intralysosomal pH in living cells and the perturbation of $\mathrm{pH}$ by various agents. Proc Natl Acad Sci USA 1978; 75: 3327-3331.

21. Huynh KK, Eskelinen EL, Scott CC, Malevanets A, Saftig P, Grinstein S. LAMP proteins are required for fusion of lysosomes with phagosomes. EMBO J 2007; 26: 313-324.

22. Chan EY. mTORC1 phosphorylates the ULK1-mAtg13-FIP200 autophagy regulatory complex. Sci Signal 2009; 2: pe51.

23. Liu P, Cheng H, Roberts TM, Zhao JJ. Targeting the phosphoinositide 3-kinase pathway in cancer. Nat Rev Drug Discov 2009; 8: 627-644.

24. Janes MR, Limon JJ, So L, Chen J, Lim RJ, Chavez MA et al. Effective and selective targeting of leukemia cells using a TORC1/2 kinase inhibitor. Nat Med 2010; 16: 205-213.

25. Chiarini F, Grimaldi C, Ricci F, Tazzari PL, Evangelisti C, Ognibene A et al. Activity of the novel dual phosphatidylinositol 3-kinase/mammalian target of rapamycin inhibitor NVPBEZ235 against T-cell acute lymphoblastic leukemia. Cancer Res 2010; 70: 8097-8107.

26. Xu WS, Parmigiani RB, Marks PA. Histone deacetylase inhibitors: molecular mechanisms of action. Oncogene 2007; 26: 5541-5552.

27. Federico M, Bagella L. Histone deacetylase inhibitors in the treatment of hematological malignancies and solid tumors. J Biomed Biotechnol 2011; 2011: 475641.

28. Rubinsztein DC, Gestwicki JE, Murphy LO, Klionsky DJ. Potential therapeutic applications of autophagy. Nat Rev Drug Discov 2007; 6: 304-312.

29. Dikic I, Johansen T, Kirkin V. Selective autophagy in cancer development and therapy. Cancer Res 2010; 70: 3431-3434

30. Janku F, McConkey DJ, Hong DS, Kurzrock R. Autophagy as a target for anticancer therapy. Nat Rev Clin Oncol 2011; 8: 528-539.

31. Shao Y, Gao Z, Marks PA, Jiang X. Apoptotic and autophagic cell death induced by histone deacetylase inhibitors. Proc Natl Acad Sci USA 2004; 101: 18030-18035.
32. Yamamoto S, Tanaka K, Sakimura R, Okada T, Nakamura T, Li Y et al. Suberoylanilide hydroxamic acid (SAHA) induces apoptosis or autophagy-associated cell death in chondrosarcoma cell lines. Anticancer Res 2008; 28: 1585-1591.

33. Ellis L, Bots M, Lindemann RK, Bolden JE, Newbold A, Cluse LA et al. The histone deacetylase inhibitors LAQ824 and LBH589 do not require death receptor signaling or a functional apoptosome to mediate tumor cell death or therapeutic efficacy. Blood 2009; 114: 380-393.

34. Lopez G, Torres K, Lev D. Autophagy blockade enhances HDAC inhibitors' pro-apoptotic effects: potential implications for the treatment of a therapeutic-resistant malignancy. Autophagy 2011; 7: 440-441.

35. Carew JS, Medina EC, Esquivel JA II, Mahalingam D, Swords R, Kelly K et al. Autophagy inhibition enhances vorinostat-induced apoptosis via ubiquitinated protein accumulation. J Cell Mol Med 2010; 14: 2448-2459.

36. Carew JS, Nawrocki ST, Kahue CN, Zhang H, Yang C, Chung L et al. Targeting autophagy augments the anticancer activity of the histone deacetylase inhibitor SAHA to overcome Bcr-Abl-mediated drug resistance. Blood 2007; 110: 313-322.

37. Hou W, Han J, Lu C, Goldstein LA, Rabinowich H. Autophagic degradation of active caspase-8: a crosstalk mechanism between autophagy and apoptosis. Autophagy 2010; 6 : $891-900$

38. Lum JJ, Bauer DE, Kong M, Harris MH, Li C, Lindsten T et al. Growth factor regulation of autophagy and cell survival in the absence of apoptosis. Cell 2005; 120: 237-248.

39. Li G, Jiang H, Chang M, Xie H, Hu L. HDAC6 alpha-tubulin deacetylase: a potential therapeutic target in neurodegenerative diseases. J Neurol Sci 2011; 304: 1-8.

40. Alers S, Loffler AS, Wesselborg S, Stork B. Role of AMPK-mTOR-Ulk1/2 in the regulation of autophagy: cross talk, shortcuts, and feedbacks. Mol Cell Biol 2012; 32: 2-11.

41. Gupta M, Ansell SM, Novak AJ, Kumar S, Kaufmann SH, Witzig TE. Inhibition of histone deacetylase overcomes rapamycin-mediated resistance in diffuse large B-cell lymphoma by inhibiting Akt signaling through mTORC2. Blood 2009; 114: 2926-2935.

Cell Death and Disease is an open-access journal published by Nature Publishing Group. This work is licensed under the Creative Commons Attribution-NonCommercial-No Derivative Works 3.0 Unported License. To view a copy of this license, visit http://creativecommons.org/licenses/by-nc-nd/3.0/

Supplementary Information accompanies this paper on Cell Death and Disease website (http://www.nature.com/cddis) 